\title{
Experimental Study on the Dynamic Caustics of the Explosive Load
}

\section{Precrack Propagation Law}

\author{
Zhengbin Yan ${ }^{1, a}$, Jinjing Zuo ${ }^{1, b}$, Yanbin Wang ${ }^{1, c}$ \\ ${ }^{1}$ School of Mechanics and Architecture Engineering, China University of Mining and Technology \\ (Beijing), Beijing, 100083, China \\ aYangzhengb2016@sina.com, 'buojinj123@sina.com, cwangyanb8@sina.com
}

Keywords: caustics; precrack; explosion

\begin{abstract}
By making use of the experimental system of explosive load digital laser dynamic caustics, it is to carry out dynamic crack analysis for the tip crack initiation and propagation process of precracks near the blast hole and study the directional crack controlled blastting mechanism, thus obtaining changes of the displacement, speed, acceleration, stress intensity factor values of the proecrack propagation in the penetration process of the precrack and blast hole. The results indicate that crack propagation displacement of the precrack direction on the premise of the penetration experiment of the precrack and blast hole is far larger than the non-precrack direction, and the caustics speckle diameter of the crack tip, the propagation speed, and the stress intensity factor value show an overall declining trend with the reach of a small peak in the middle and later period of the propagation process.
\end{abstract}

\section{Introduction}

To reduce the dense radial cracks produced near the blast hole and allow crack propagation to be fuly realized in the controlled blasting of sinking and driving engineering, the engineering practice has shown that cutting and sewing cartridge bags can help to achieve postiive effects on the peripheral molding quality of the tunnel[1-3]. The crack mechanics act of rocks in dynamic load is very complicated, and the interaction between the stress wave and the crack is hard to be explained. Therefore, testing the crack mechanics is a key means to study the dynamic crack mechanics of rocks[4]. Flash ranging of crack mechanics is an optimal test method in dynamic crack researches, and the dynamic caustics test method is with high precision and convenient calculation. The characteristic length of caustics speckle provides a quantitative measurement for the stress intesnity factor on the crack tip, which has been widely applied to the dynamic crack mechanics study. P. Manogg [5] initailly proposed to apply the caustics method to crack mechanics researches. Domestic scholars Wu Xiang [6] and Su Xianji [7] carried out the study on caustics method respectivley and studied some static crack problems with this method. In 1971, P.S. Theocaris [8] promoted the caustics method from the theory level to the reflection situation, and he studied the plasticity area size near the crack tip, the crack tip position and stress intensity factor, and he also measured characteristics of the crack tip stress field of the crack tip in the condition of reflective caustics of metal materials. Li Zheng et al. [9] measured the dynamic fracture toughness of set cement by using the reflective caustics method. Duan Zili et al. [10-12] measured the open amount of the crack tip of metal samples with the method of reflective caustics and fake caustics. Yang 
Renshu et al. [13] condcuted the transmittance dynamic caustics test under explosive load by using an organic glass model, and they studied the influence of closed and open joints under the explosive load on the cutting and sewing cartridge bag crack controlled blasting crack propagation law. The corresponding failure mode, dynamic stress intensity factor and crack propagation speed changes are analyzed as well. Xiao Tongshe et al. [14] used the dynamic caustics to simulate microscomic act of explosive crack propagation in rocks with joints, thus summarizing the general law of explosive crack propagtion passing through the joints.

The explosive load reflective dynamic caustics test system is adopted this time. By making use of the organic glass (PMMA) model, the flaw-containing medium crack propagation test under the effect of explosive stress wave is carried out, and the dynamic caustics image of flaw-containing medium is obtained. The mechanical parameters of flaw-containing medium crack propagation is analyzed quantitatively, the relationship of crack propagation displacement, speed, dynamic stress intensity factor and dynamic crack propagation time changes is determined, and the crack propagation mechanism is discussed. These researches can provide theoretical basis for the design of blasting parameters controlled by flaw-containing fracture rock cracks.

\section{Experiment details}

\section{Explosion loading digital laser caustics test}

Caustics method [15] is used to convert the complex degeneration state of the stress concentration region in one object to the simple and clear shadow optical graphics through the mapping relation of geometrical optics, as shown in Fig.1.

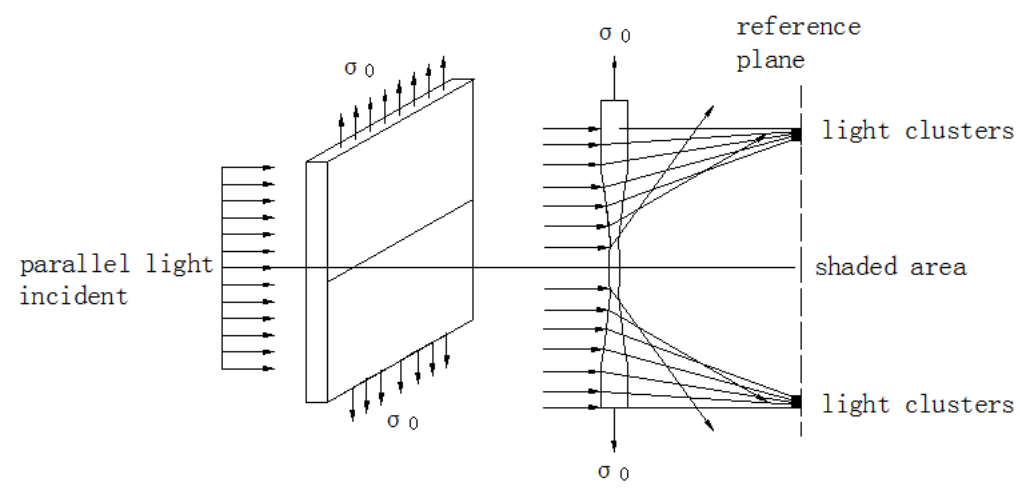

Fig. 1. Caustics image

\section{Test system}

The new digital laser dynamic caustics testing system is composed of laser device, beam expander, field lens assembly, digital high-speed camera and other. Laser device emits the continuous stable bright light waves, which are changed to parallel incident lights on the surface of specimen through the beam expander and field Lens 1, the light beams after deflection aggregate into the high-speed camera lens, the photographing and recording speed is varied to photograph the change of light intensity at the reference plane, record the dynamic caustics and obtain the image of digital shadow spots. This system can be used to make photomechanics analysis of the testing process of blasting, impact and other dynamic fracture tests, and the optical path system is simple, user-friendly and easy to observe with reduced experimental cost to increase the testing precision and success rate. Fig. 2 gives the optical path of transmission type caustics testing system. 


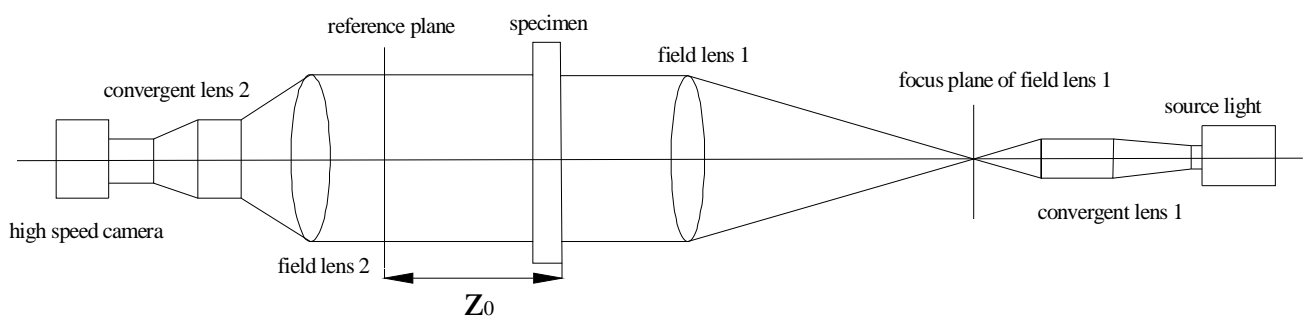

Fig. 2. The optical path of transmission type caustics test system

\section{Test data processing}

(1) Determination of crack tip displacement

The position of instantaneous crack tip can be precisely measured according to the high-speed photographic image, after being converted to the scale of image to object, the displacement of crack tip can be obtained.

(2) Determination of velocity $v$ and accelerated speed $a$ of crack propagation

The average velocity of crack propagation in this time interval can be obtained by the displacement of crack tip at each instantaneous moment acquired from part (1); the differential value of crack length in the adjacent two images, divided by the time interval between two images.

To reduce the degree of data dispersion, the average velocity of crack propagation was fitted to the quintic polynomial [16] of time $t$ for the analysis of the acceleration of crack propagation:

$$
v(t)=\sum_{i=0}^{5} v_{i} t^{i}
$$

Wherein, $v(t)$ is the fitting average velocity, the coefficient $v_{i}$ is obtained through the least square method. Thus, the accelerated speed $a$ of crack propagation is obtained by solving the first order derivative to the curve $v(t)$.

(3) Dynamic stress intensity factor (DSIF)

The explosion pressure pulse in the explosion stress field mainly transmits in the form of two body waves: expansion wave and shear wave. Subject to these two types of waves, the normal stress and shear stress are produced on any point in the medium, which means that the explosion stress field is complex, so the crack in the medium can be treated as complex crack. With a view to the dynamic caustics testing method, according to the testing principle, the DSIF of crack tip can be expressed as:

$$
\begin{aligned}
& K_{I}^{d}=\frac{2 \sqrt{2 \pi}}{3\left|c_{t}\right| d z_{0}}\left[\frac{3}{10 \sin \left(\frac{2}{5} \pi\right)}\right]^{\frac{5}{2}}\left(\frac{D_{t}}{\delta_{t}}\right)^{\frac{5}{2}} \\
& K_{I I}^{d}=\mu K_{I}^{d}
\end{aligned}
$$

Wherein, $D_{t}$ is the longitudinal diameter of shadow spot; $z_{0}$ is the distanced from the reference plane to the specimen plane, in consideration of the effect of the plastic degeneration of crack tip, 
this test chose $z_{0}=900 \mathrm{~mm} ; c_{t}$ is the stress optical constant of material, $c_{t}=0.88 \times 10^{-10} \mathrm{~m}^{2} / \mathrm{N} ; \quad d$ is the valid thickness of specimen, as to the transparent PMMA plate, the valid thickness of plate is the actual thickness of plate; $K_{I}^{d}, K_{I I}^{d}$ is I, II mode DSIF at the complex crack tip subject to the dynamic load; $\mu$ is the scale factor of stress intensity factor; $\delta_{t}$ is the correction factor as a result of crack propagation velocity, with the crack propagation velocity with actual meaning, the value equals to 1 .

\section{Model design}

To study the interaction between the explosive load and the precrack, premade a fissure that penetrating through the entire plate on both sides of the blast hole of the organic glass plate, the length of which should be $2 \mathrm{~cm}$ and the blast hole diameter should be $8 \mathrm{~mm}$. The blast hole and the premade fissure are on the same line, and the blast hole wall and the remade fissure penetrate through it, as shown in the figure. The blast hole contains $150 \mathrm{mg}$ lead azide single-compound explosive.

\section{Experimental process and result analysis}

\section{Description of the experimental phenomenon}

Fig. 3 shows the figure of the tunnel model contrast before and after the experiment. As can be seen from Figure 3b, under the effect of the explosive dynamic load, the precrack shows the propagation along the crack tip, and the crack propagation at the symmetrical position is basically consistent but not exactly the same. This is caused by the uneven distribution of the experimental explosive and impurities as well as the processing errors of the plate. The expanded displacement of the main crack 1 is $7.3 \mathrm{~cm}$, that of the main crack 2 is $7.7 \mathrm{~cm}$, that of the wing crack 1 is $2.3 \mathrm{~cm}$, and that of the wing crack 2 is $2.8 \mathrm{~cm}$. The propagation length of the main crack is larger than that of the wing crack. The crack propagation is sorted out as shown in Table 1:

Table 1 Final propagation displacement of the crack

\begin{tabular}{cc}
\hline No. & Displacement/ cm \\
\hline Main crack 1 & 7.3 \\
Main crack 2 & 7.7 \\
Wing crack 1 & 2.3 \\
Wing crack 2 & 2.8 \\
\hline
\end{tabular}

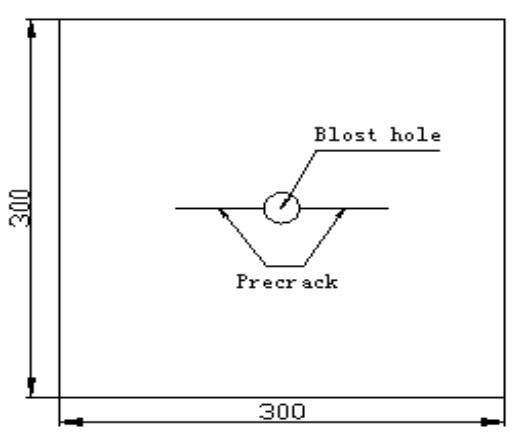

(a) Before the experiment

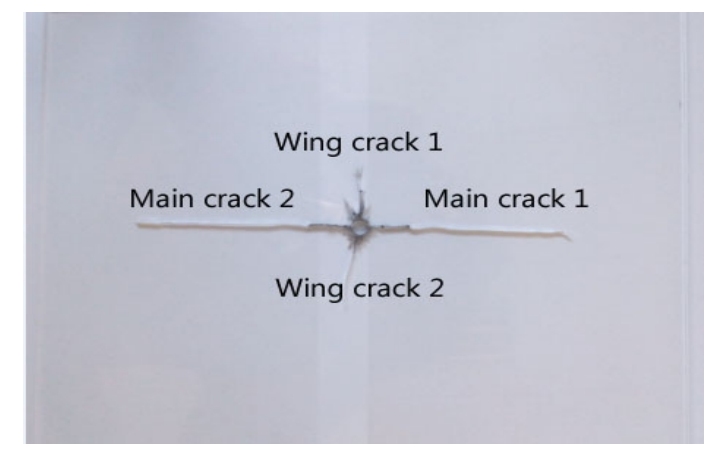

(b) After the experiment

Fig.3. Figures of the contrast before and after the experiment 


\section{Study and analysis on the crack propagation displacement}

Due to limitation of the vision field, the crack propagation image cannot be seen fully, and only the propagation of the right crack 1 can be observed.

Fig. 4 shows the curve of the crack tip displacement. When $t=40 \mu$ s, the crack starts to expand, and the displacement curve starts to increase gradually. When $t=50 \mu \mathrm{s}$, the displacement curve remains to grow at a certain rate. When $\mathrm{t}=110 \mu \mathrm{s}$, the growth of the displacement curve starts to decline slightly. When $t=150 \mu \mathrm{s}$, the displacement curve grows again at a certain rate. And when $\mathrm{t}=230 \mu \mathrm{s}$, the displacement growth rate will drop till the crack propagation ends. In the whole process, the displacement propagation does not rise at certain slope, during which, the time curve rate is smaller than the curve rate of the first half section and second half section for a while. The reason for this is that the crack propagation can be divided into two parts. For the first half part, the displacement propagation is motivated by the stress wave, and for the second half part, it is caused by the explosive products. After the explosive blasts in the crack propagation process of the first half part, the shock wave working on the blast hole wall will soon be reduced to the stress wave in the medium, and the stress wave will gather in the crack tip, making the crack tip produce stress concentration until it meets conditions for the crack initiation. Driven by the stress wave, cracks will expand forward. Due to the energy consumption in the crack propagation process, the displacement slope will drop in the middle section of crack propagation. At this time, products caused by the blast will reach the tip of the crack propagation along the fissure, thus making up for the energy loss. Therefore, the driving force of the second half part of crack propagation is caused by blast products which provide energies for the crack tip until the latter stops propagation. There are many researches about the crack propagation mechanism, but no unified theoretical support is available. The research result of this experiment is also limited to this research background.

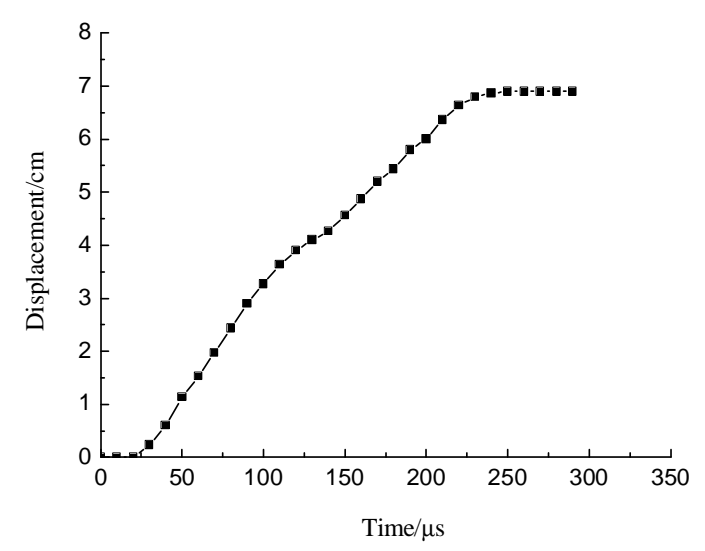

Fig. 4. Curve of the crack propagation displacement

\section{Study and analysis on the dynamic caustics of crack propagation}

Fig. 5 shows the series of caustics speckle image of crack propagation, and Fig. 6 shows the time-distance curve of the diameter size ofcaustics speckles at the crack tip. The tip caustics speckle features are analyzed by combining the two. When $t=30 \mu \mathrm{s}$, the caustics plate starts to appear with the diameter of $1.33 \mathrm{~mm}$. When $\mathrm{t}=50 \mu \mathrm{s}$, the crack has started to expand with certain slope. At this time, the diameter of the caustics speckle is $5.33 \mathrm{~mm}$. When $\mathrm{t}=70 \mu \mathrm{s}$, the real-virtual caustics speckles start to appear at the crack tip. It is caused by the production of puff wave ( $\mathrm{P}$ wave) and shear wave ( $\mathrm{S}$ wave) after the explosive blasts. The speed of $\mathrm{P}$ wave is almost 1.7 times that of $\mathrm{S}$ wave. In the transmission process, they are mutually separated. In the plane problem, $\mathrm{P}$ wave spreads out by taking the explosion source as the center, while the shape of $S$ wave in the 
transmission process is rather disordered. Their encircled reflection and scattering at the crack tip leads to the complicated stress state of the crack tip. At this time, the diameter peak of the caustics speckle is $5.66 \mathrm{~mm}$. When $\mathrm{t}=70-120 \mu \mathrm{s}$, the crack propagation is rather stable, and the caustics speckle diameter only fluctuates within a small range. When $t=140 \mu \mathrm{s}$, the first minimum caustics speckle diameter of $3.66 \mathrm{~mm}$ can be shown. When $\mathrm{t}=170 \mu \mathrm{s}$, the second peak diameter of the caustics speckle can be seen, which is $5.33 \mathrm{~mm}$. When $\mathrm{t}=170-220 \mu \mathrm{s}$, the diameter of the caustics speckle fluctuates within a small range. When the crack propagation stops, the caustics speckle will disappear. When $\mathrm{t}=70 \mu \mathrm{s}$, explosive products will start to expand out through the cutting gap, which indicates that no explosive product has been generated in the earlier stage as the driving force for crack propagation. Through the overall analysis of the caustics speckle diameter figure, it can be seen that the whole crack tip propagation process is rather steady, except for the bottom shown up for a while in the process. The reasons of this have been explained above.

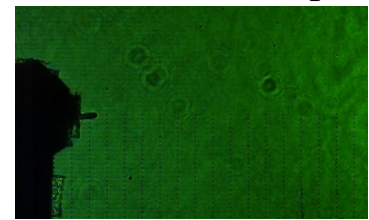

$\mathrm{t}=0 \mu \mathrm{s}$

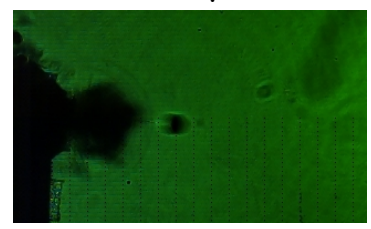

$\mathrm{t}=90 \mu \mathrm{s}$

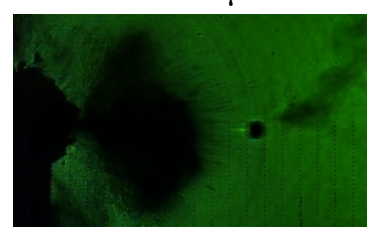

$\mathrm{t}=170 \mu \mathrm{s}$

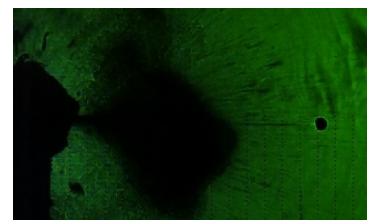

$\mathrm{t}=250 \mu \mathrm{s}$

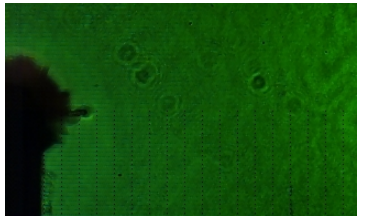

$\mathrm{t}=30 \mu \mathrm{s}$

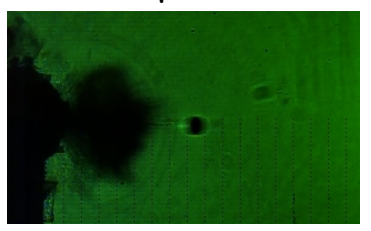

$\mathrm{t}=110 \mu \mathrm{s}$

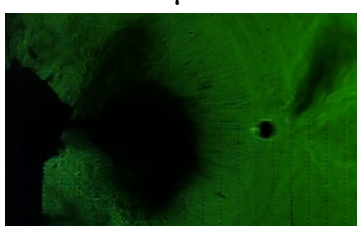

$\mathrm{t}=190 \mu \mathrm{s}$

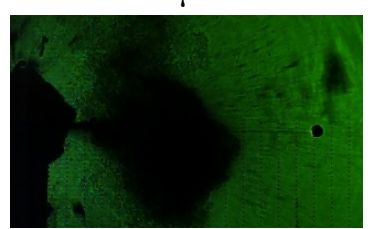

$\mathrm{t}=270 \mu \mathrm{s}$

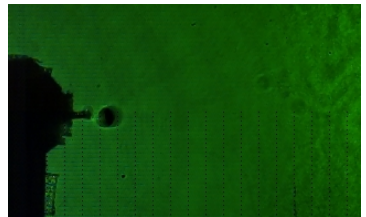

$\mathrm{t}=50 \mu \mathrm{s}$

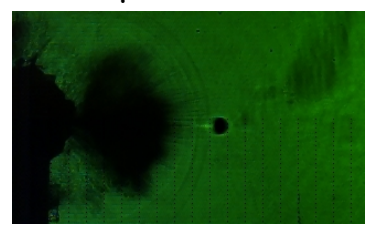

$\mathrm{t}=130 \mu \mathrm{s}$

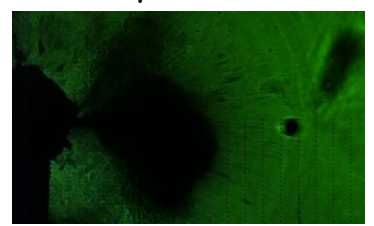

$\mathrm{t}=210 \mu \mathrm{s}$

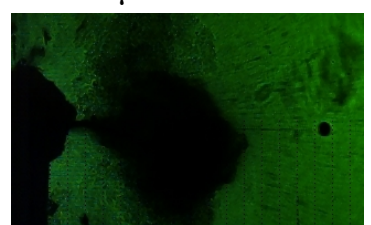

$\mathrm{t}=290 \mu \mathrm{s}$

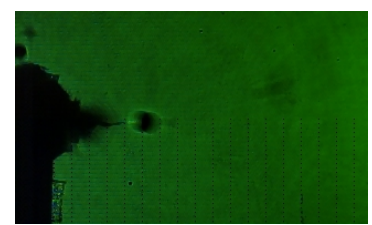

$\mathrm{t}=70 \mu \mathrm{s}$

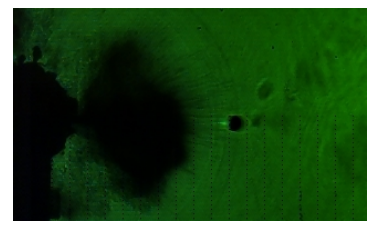

$\mathrm{t}=150 \mu \mathrm{s}$

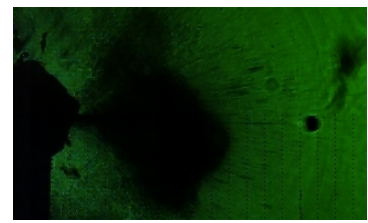

$\mathrm{t}=230 \mu \mathrm{s}$

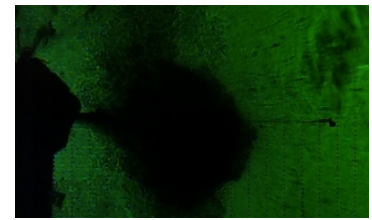

$\mathrm{t}=310 \mu \mathrm{s}$

Fig. 5.Series of casutics speckle iamge of the crack propagation

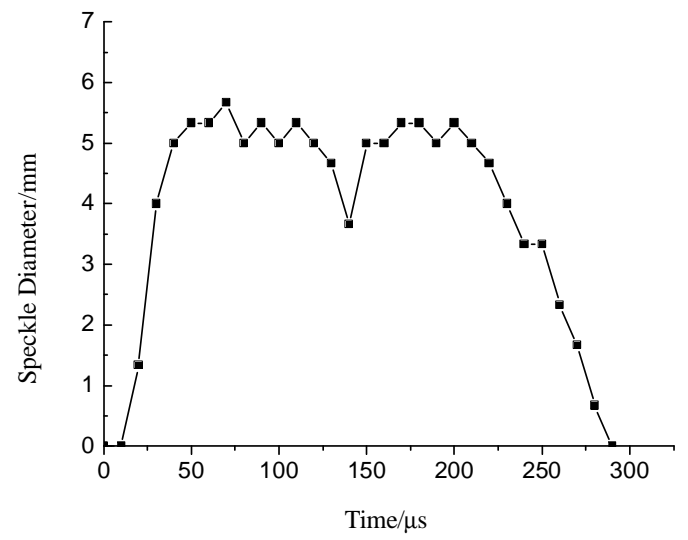

Fig.6. Time-distance curve of the caustics speckle size at the crack tip 


\section{Study and analysis on the speed and acceleration of crack propagation}

Fig.7 shows the crack propagation speed and acceleration speed. It can be obtained from the analysis of the speed curve: when $t=30 \mu \mathrm{s}$, cracks start to expand. When $t=50 \mu \mathrm{s}$, it reaches the peak speed of $533 \mathrm{~m} / \mathrm{s}$, followed by the speed fluctuation. When $\mathrm{t}=140 \mu \mathrm{s}$, the minimum value of speed appears, which is $166 \mathrm{~m} / \mathrm{s}$. When $\mathrm{t}=150-220 \mu \mathrm{s}$, the speed fluctuates and its mean value is $280 \mathrm{~m} / \mathrm{s}$. When $\mathrm{t}=260 \mu \mathrm{s}$, the cracking stops and the speed is $0 \mathrm{~m} / \mathrm{s}$. Through the analysis of the acceleration curve, it can be seen: when $\mathrm{t}=20-40 \mu \mathrm{s}$, the acceleration is always on the increase stage, suggesting the speed increase is larger and larger. When $\mathrm{t}=40 \mu \mathrm{s}$, the acceleration reaches the first peak of $18.33 \mathrm{~m} / \mathrm{s}^{2}$. When $\mathrm{t}=50-70 \mu \mathrm{s}$, the acceleration starts to reduce to $0 \mathrm{~m} / \mathrm{s}^{2}$ or lower, and the first minimum value $-4.993 \mathrm{~m} / \mathrm{s}^{2}$ appears. When $\mathrm{t}=80-130 \mu \mathrm{s}$, the acceleration decreases after the first increase. When $\mathrm{t}=130 \mu \mathrm{s}$, the second minimum acceleration shows, which is $-8.33 \mathrm{~m} / \mathrm{s}$. When $\mathrm{t}=140-160 \mu \mathrm{s}$, the acceleration comes to the rise stage, and when $\mathrm{t}=160 \mu \mathrm{s}$, the peak $6.66 \mathrm{~m} / \mathrm{s}$ appears. After that, it basically remains at about $0 \mathrm{~m} / \mathrm{s}$ until the final crack stops propagation. The change of the acceleration curve is exactly consistent with that of the speed, and the speed curve is also consistent with the displacement curve.

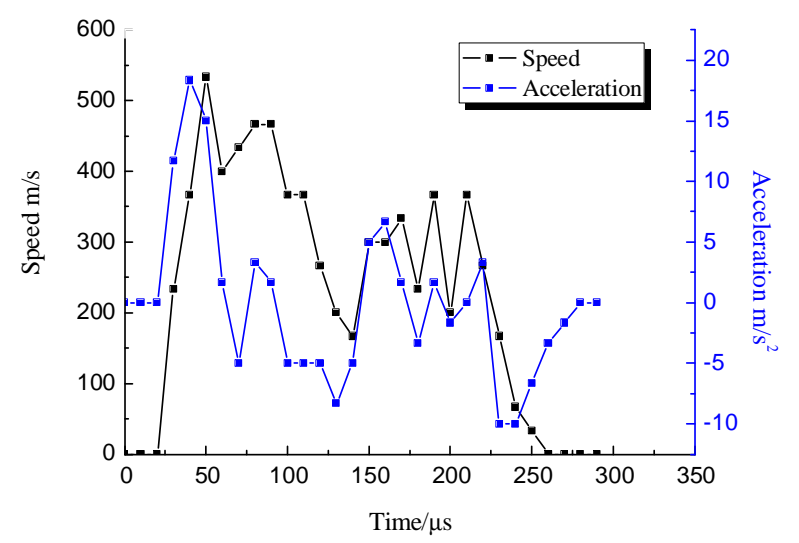

Fig. 7. Time-distance curve of the speed and acceleration at the crack tip

\section{Study and analysis on the crack propagation stress intensity factor}

Fig. 8 shows the time-distance curve of the stress intensity factor at the crack tip. The time-distance curve of the stress intensity factor is consistent with the time-distance curve of the caustics speckle size changes. When $\mathrm{t}=20 \mu \mathrm{s}$, caustics speckles start to appear. It needs to be noticed that caustics speckles appear earlier than crack propagation, which indicates there is a section of energy accumulation process at the crack tip before the crack initiation. When $t=70 \mu \mathrm{s}$, the first peak of $K_{1}^{d}$ is $0.52 M N \cdot m^{-3 / 2}$. Due to the effect of $\mathrm{S}$ wave, the value of stress intensity factor fluctuates within certain ranges. When $\mathrm{t}=140 \mu \mathrm{s}$, the bottom value of stress intensity factor appears, which is $1.17 \mathrm{MN} \cdot \mathrm{m}^{-3 / 2}$. After that, the stress intensity factor value fluctuates within some certain range until the crack stops propagation. The disappearance of caustics speckles is later than the cracking stop, indicating that caustics speckles do not disappear immediately after the cracking stops. 


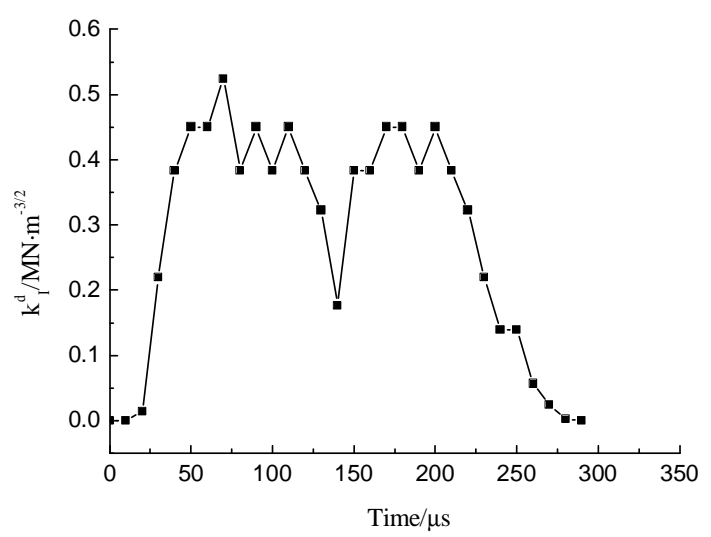

Fig. 8. Time-distance curve of the stress intensity factor at the crack tip

\section{Conclusions}

(1) In the connection direciton of the blast hole and crack, the crack propagation length is larger than the propagation length of the wing crack. The maximum crack propagatio length is $7.7 \mathrm{~cm}$, which is 3.3 times that of the wing crack.

(2) The entire crack propagation stage can be divided into two parts. The driving force of displacement propagation in the first half part is caused by the stress wave, and that in the second half part is caused by explosive products.

(3) There is a bottom value for the crack speedand stress intensity factor in the middle phase of the crack propagation. Due to the effect of $S$ wave, its value has always been fluctuating.

\section{Reference}

[1] Yuming Zhang, Yongfeng Yuan, Qi Zhang. The slit-charge breaking rock mechanism and application[J].Explosive Materials, 2001, 30(5):5-8.

[2] Yongqi Yang, Qiankun Jin, Renshu Yang, et al. A new technology of directional fracture blasting in rock drift[J]. Engineering Blasting , $1995,1(1): 8-10$.

[3] Zhonghua Tang, Zhicheng Zhang, Kaiwei Xiang. The mechanism of lancing cartridge blasting[J].Yunnan Metallurgy , 1998 , 27(4):7-11.

[4] Zaihua Liu, De Jie, Yuanhan Wang, et al. Fracture dynamics engineering[M]. Wuhan: Huazhong University Press, 1996: 281-300.

[5] MANOGG P. International conference on the physics of non-crystalline solids[M]. Netherlands: [s. n.], 1964: 481-490.

[6] Xiang Wu, Huaitang Yang. Application of caustics method in fracture (measuring SIF )[J]. Acta Mechanica Solida Sinica, 1984, (2): 299-308.

[7] Xianji Sun, Cheng Liu. Summary of caustics experiment method[J]. Journal of Experimental Mechanics, 1987,2(2): 1-27.

[8] THEOCARIS P S. Reflected shadow method for the study of constrained zones in cracked plates[J]. Applied Optics, 1971, 10 (10): 2240-2247.

[9] Zheng $\mathrm{Li}$, Xianji $\mathrm{Su}, \mathrm{Bin} \mathrm{Fu}$. Dertermination of dynamic fracture toughness for cement 
block[J].Mechanics in Engineering, 1999, 21(1): 41-44.

[10] Zili Duan. Measuring the crack tip COD of metal specimen by caustics[J]. Journal of Huazhong University of Science and Technology, 1995, 23(5): 117-122.

[11] Zili Duan. Experimental research of unstable crack growth track and pseudo-caustics[J]. Acta Mechanica Solida Sinica, 1995, 16(3): 269-273.

[12] Zili Duan. Experimental research of plane angle unstable crack growth track by caustics method[J].Journal of Huazhong University of Science and Technology, 1995, 23(3): 94-99.

[13] Renshu Yang, Zhongwen Yue, Tongshe Xiao, et al.Dynamic caustics experiment on crack propagation of jointed medium fracture with controlled blasting[J].Chinese Journal of Rock Mechanics and Engineering,2008, 27(2): 244-250.

[14] Tongshe Xiao, Renshu Yang. Dynamic caustics model experiment of blasting crack developing on sandwich rock[J]. Explosion and Shock Waves, 2006,27(2): 159-164.

[15] Renshu Yang , Yanbing Wang , Liyun Yang, et al. Dynamic caustics experimental study on crack propagation in two borehole cut blasting[J]. Journal of China University of Mining and Technology , 2012 , 41(6) : 868-872.

[16] Liyun Yang, Renshu Yang. Zhongwen Yun, et al. Digital laser dynamic caustics experimental system[P]. Chinese Patent: 201110366309.9,2012-07-04. 